\title{
Synthesizing of models for identification of teletraffic Markov chains by artificial neural networks and decision tree method
}

\author{
Ivelina Stefanova Balabanova*, Georgi Ivanov Georgiev*, \\ Stanimir Michaylov Sadinov*, Stela Savova Kostadinova**
}

\begin{abstract}
Imitation modelling processes of telegraphic systems on the Markov chains with unlimited and limited queues were made. For this purpose, the Java modeling tool simulation environment is used. With a fixed number of client stations and a number of system users, data are accumulated about the telegraphic system parameters as: customer ID, arrival time, server ID and exit system. Artificial neural networks (ANN) with backpropagation algorithm and decision tree (DT) method for identification of the studied Markov chains in MATLAB were applied. Training of the structural identification models to determine of the membership of the obtained parameters in telegraphic simulation to both unlimited and limited systems was carried out. The results of the training and synthesis of ANN and DT models are presented. Sufficient results have been obtained for telegraphic identification confirming the successful application of the proposed synthesized classification models, approximately $91 \%$ for DT and $99.2 \%$ for ANN.
\end{abstract}

K e y w o r d s: Markov chain, artificial neural network, decision tree, synthesized models, telegraphic identification

\section{Introduction}

The relationship between artificial neural networks (ANN) and Markov chains in the field of communication telegraphic systems is most often expressed in the combination of ANN architectures and Markov's hidden models (NMMs) in general technical methods and approaches for modeling and data mining from different types of data sets. An example of this is their use in algorithms designed to distinguish individual sound templates to improve the quality of mobile and embedded speech recognition systems. Successful neural network LVQ (learning vector quantization) and neural network RBF (radial bias function) and dynamic multi-layer perceptrons tests were conducted $[1,2]$. The development of deep Markov neural networks (DMNNs) - HMMs and recursive neural networks, is effectively used to analyze of information symbol sequences to predict topics and sentiments in dialogues [3].

The subject of the research is also the process of generating of complex neural structures based on recurrent neural networks, used to modeling of telegraphic states and Markov's chain transitions. In these cases, the networks are composed of an attractor network with a stabilizing recurrent connection (memory network), a noise network which stores the transitions between the attractors occurring uniformly at random and the network is another attractor network which receives input from both memory and noise networks[4].
In its specificity, the DT method is designed for qualitative and quantitative data analysis (data classification). In other studies is demonstrated its use in training and improving Markov network structures by developing algorithms that implement logistic regression training to transform the specified networks into probabilistic decision trees (DTSL structures). The main advantages of DTSL are the speed of training and the cutting of complex correlations between multiple variables $[5,6]$.

The processes of change of the classical decision tree learning algorithms by Bayesian algorithmic procedures using Markov chain Monte Carlo (MCMC) are discussed in other sources [7]. Fact is the creation of the hidden tree Markov network (HTN) - a neural hybrid architecture which combines the power of generative models for trees with the incremental and discriminative learning capabilities of the neural network. In this way a greater depth of unfolding of the generative models on the input structures is guaranteed [8].

Other studies are focused on the speed-up simulation of parameters and teletraffic models for RESTART method with implementation of LRE in teletraffic systems of network of queues and $5 \mathrm{G}$ networks $[9,10]$.

The paper explores potential applications of the ANN and DT methods other than the classic ones in the analysis of HMM, adapting their capabilities to innovative object-oriented classification tasks for investigation of Markov chains. Algorithmic approaches to synthesizing and selecting of models for identification of telegraphic systems with Markov processes based on artificial intel-

* Technical University of Gabrovo, Bulgaria; Faculty of Electrical Engineering and Electronics, Department of Communications Equipment and Technologies; H. Dimitar Str.4, 5300, Gabrovo city, Bulgaria, ivstoeva@abv.bg, givanow@abv.bg, sadinov.tc@abv.bg,** Technical University of Varna, Bulgaria; Faculty of Electronics, Department of Communications Engineering and Technologies; Studentska Str. 1, 9010, Varna, Bulgaria, stela.kostadinova@tu-varna.bg 
ligence and graphical method for alternative choice between interrelated solutions are introduced.

\section{Modelling of Markov chains-experimental data}

Using a Java modeling chain Markov (JMCH) simulator, part of the Java modeling tool, we were modelling the following teletraffic systems:

- $\mathrm{M} / \mathrm{M} / \mathrm{c}$ : system with different number of $c$ servers and unlimited queue size,

- $\mathrm{M} / \mathrm{M} / \mathrm{c} / \mathrm{k}$ : system with different number of servers $c$ and limited queue size $k$.

The modelling telegraphic processes are performed at a constant number of servers $c=25$ and a corresponding number $k=1000$ of system users. The following simulation parameters are set:

- Average arrival rate (lambda) in customers/second average speed of arrival in the system,

- Average service time $S$ in seconds average service time; at levels 0.5 and 1.9 for system $\mathrm{M} / \mathrm{M} / 25$ and relevant parameters:

- Average arrival rate $(\lambda)$ - average speed of arrival in the system,

- Average service time $S$ - average service time;

- Maximal station capacity $k$ maximum capacity of the queue, at levels $0.5,1.9$ and 26 for system M/M/25/k. Data sets with output parameters were obtained:

- Customer ID user ID;

- Arrival Time (s) time to stay in the system,

- Server ID server ID,

- Exit system (s) the exit time, as the data being stored in csv files.

On the basis of the obtained experimental data, a task in the field of study "telegraphic communication systems for batch data transmission" for parameter identification of $\mathrm{M} / \mathrm{M} / 25$ and $\mathrm{M} / \mathrm{M} / 25 / \mathrm{k}$ chains is formed. It is envisaged the decision task will be done in MATLAB environment. The data sets with parameter values included - customer ID; arrival time; server ID and exit system (determined as input parameters for classification models), with 2000 samples, respectively 1000 samples for each Markov chain $\mathrm{M} / \mathrm{M} / 25$ and $\mathrm{M} / \mathrm{M} / 25 / \mathrm{k}$ (defined as output classification groups). For training and testing of the created ANN and DT classification models was taken a dataset of 602 samples (301 samples for each target group) with teletraffic parameters - customer ID; arrival time; server ID and exit system. The purpose of recognition and classification processes is to be determined the membership of the telegraphic parameters to the relevant Markov chain.

\section{Synthesis of Markov chain identification model based on artificial neural networks}

In the initial stage of artificial neural networks with back propagation are used classical three-layer architectures (one input layer, one hidden layer and one output layer). Structures with different hidden neurons in the range of 10 to 25 were studied. The obtained results for the classification accuracy turned out to be quite low for successful teletraffic identification. For that reason, the investigated neural structures were modified by adding more hidden layers. More than 3 layers were not added because the large neural networks require more computer resources and a significant data processing time. The neural networks with three hidden layers had worse identification performance. The best metrics were achieved in neural models with two hidden layers.

The training procedures for selecting of 4-layer artificial neural networks to identify chains $\mathrm{M} / \mathrm{M} / 25$ and $\mathrm{M} / \mathrm{M} / 25 / \mathrm{k}$ were made by use of $60 \%$ (361 samples) from the input dataset (602 samples). The remaining 40\% (241 samples) of dataset are allocated for validation - $20 \%$ and $20 \%$ for testing for the best selected neural architecture. The training process is completed by LevenbergMarquardt algorithm (other potential algorithm which can be used is scaled conjugate gradient algorithm) and the followed parameters - epochs: 1000 , show: 25 , goal: 0.01 , lr.: 0.05 and min_grad: $10^{-5}$.

In Tab. 1 are presented results of neural training, which contain data on the accepted quality criteria for telegtraffic system recognition, respectively mean squared error and classification accuracy.

Table 1. Results from training of multilayer artificial neural networks at recognition and classification of $\mathrm{M} / \mathrm{M} / 25$ and $\mathrm{M} / \mathrm{M} / 25 / \mathrm{k}$ chains

\begin{tabular}{|c|c|c|c|c|c|}
\hline No. & $\begin{array}{l}\text { Hidden } \\
\text { layers } \\
1 \text { and } 2\end{array}$ & Accuracy & No. & $\begin{array}{l}\text { Hidden } \\
\text { layers } \\
1 \text { and } 2\end{array}$ & Accuracy \\
\hline 1 & 10 & 95.8 & 9 & 18 & 96.7 \\
\hline 2 & 11 & 88.3 & 10 & 19 & 95.8 \\
\hline 3 & 12 & 97.5 & 11 & 20 & 98.3 \\
\hline 4 & 13 & 99.2 & 12 & 21 & 95.8 \\
\hline 5 & 14 & 99.2 & 13 & 22 & 97.5 \\
\hline 6 & 15 & 99.0 & 14 & 23 & 91.7 \\
\hline 7 & 16 & 41.7 & 15 & 24 & 97.5 \\
\hline 8 & 17 & 95.0 & 16 & 25 & 94.2 \\
\hline
\end{tabular}

input: customer ID, arrival time,server ID, exit system

output type: $\mathrm{M} / \mathrm{M} / 25 ; \mathrm{M} / \mathrm{M} / 25 / \mathrm{k}$

According to the presented results, the lowest accuracy of $41.7 \%$ is achieved at 16 neurons and the highest $99.2 \%$ is obtained at 13 and 14 neurons in the two hidden layers, respectively. The minimum and maximum mean squared errors are equal to 0.0035 and 0.3107 . In architecture with 13 neurons the MSE is 0.0191 - a lower error than that at 14 neurons, therefore as a better decision for teletraffic classification the neural architecture with 14 hidden neurons in the hidden layers is determined. 


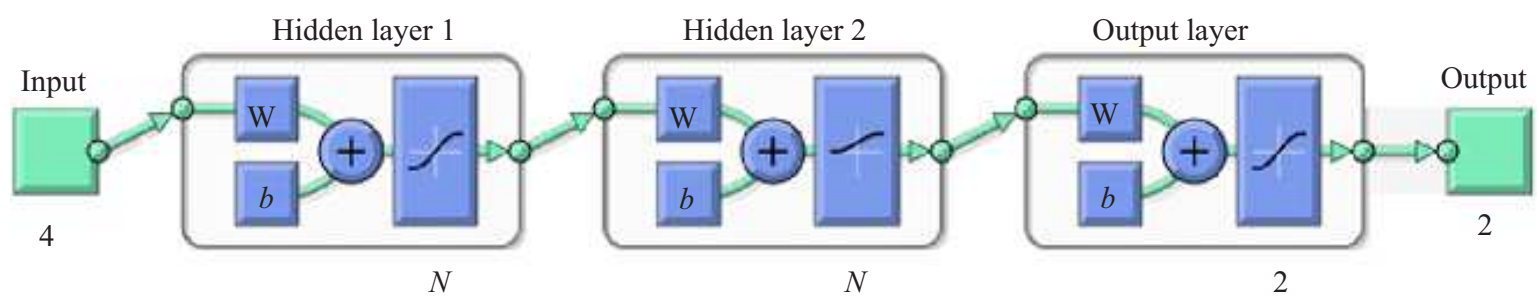

Fig. 1. Architectures of 4-layer artificial neural networks: best results with $N=14$, worst with $N=16$
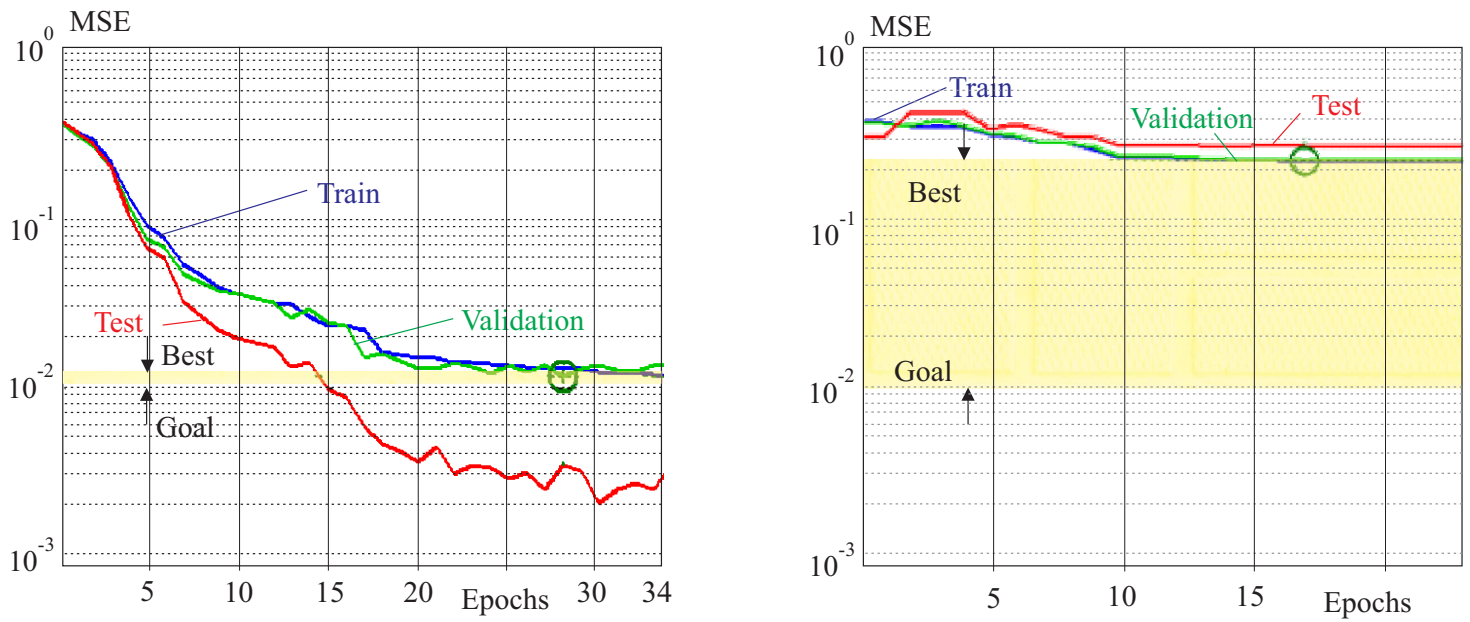

Fig. 2. Performance of 4-layer artificial neural networks at teletraffic classification: (a) - best case - validation of performance is 0.01164, at epoch 23 and (b) - worst case - the validation of performance is 0.25064 , at epoch 17

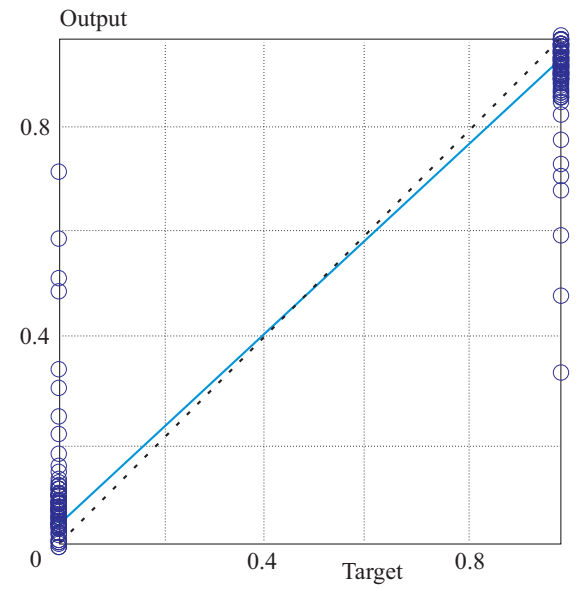

Fig. 3. Linear regression dependence at training of ANN model

Table 2. Confusion matrix for synthesized network for teletraffic system identification

\begin{tabular}{lccc}
\hline & \multicolumn{3}{c}{ Target class } \\
\hline Output & 63 & 0 & $100 \%$ \\
class 1 & $52.5 \%$ & $0.0 \%$ & $0.0 \%$ \\
\hline Output & 1 & 56 & $98.2 \%$ \\
class 2 & $0.8 \%$ & $46.7 \%$ & $1.8 \%$ \\
\hline & $98.4 \%$ & $100 \%$ & $99.2 \%$ \\
& $1.6 \%$ & $0.0 \%$ & $0.8 \%$ \\
\hline
\end{tabular}

Figure 1 shows neural network architecture of the Markov chain classification. The architecture is consist of one input layer (the layer of input variables), two hidden layers with tan-sigmoid and log-sigmoid transfer functions and one output layer with tan-sigmoid function of activation.

The performance of artificial neural networks with the best and worst performance in telegraphic classification is presented in Fig. 2. The learning processes continued in 34 training cycles for the first and 23 iterations for the second created neural models. The best validation performances 0.011641 and 0.25064 at the 28 -th and 17 -th epochs were achieved.

Figure 3 illustrates the linear regression dependence in training of the synthesized 4-layer neural architecture". The linear regressions between the target values of the model (for the first class - values "1" and "0" at Output neuron 1 and Output neuron 2; for the second class - values "0" and "1", respectively for Output neuron 1 and Output neuron 2 of ANN architecture) and the output calculated results by neuron model. Very good values of the regression coefficients $R_{i}$ were obtained, respectively $R=0.97953, R=0.99141$ and $R=0.97568$ at train, validation and test procedures. The high levels of $R_{i}$ confirm a very good approximation and linear relationship between target and output values of the ANN.

Table 2 shows the distribution of the classified test samples at teletraffic system identification based on the generated confusion matrix. Correctly classified are 63 samples from class 1 and 56 samples from class 2 as 1 sample about //25 system is incorrectly determined to the second Markov chain $\mathrm{M} / \mathrm{M} / 25 / \mathrm{k}$. Classification parameters are as follows - Sensitivity $98.4 \%$ for output 
382 I. S. Balabanova, G. I. Georgiev, S. M. Sadinov, S. S. Kostadinova: SYNTHESIZING OF MODELS FOR IDENTIFICATION OF ...

Table 3. Results from training of multilayer artificial neural networks at recognition and classification of $\mathrm{M} / \mathrm{M} / 25$ and $\mathrm{M} / \mathrm{M} / 25 / \mathrm{k}$ chains

\begin{tabular}{|c|c|c|c|c|c|c|}
\hline \multirow{2}{*}{$\begin{array}{l}\text { Pruning } \\
\text { level }\end{array}$} & \multirow[b]{2}{*}{$\begin{array}{c}\text { Number of } \\
\text { nodes }\end{array}$} & \multicolumn{2}{|c|}{ Resubstitution } & \multicolumn{2}{|c|}{ Cross-validation } & \multirow{2}{*}{$\begin{array}{l}\text { Approximately } \\
\text { expected accuracy } \\
\text { in classifying } \\
\text { new data }(\%)\end{array}$} \\
\hline & & Error & $\begin{array}{c}\text { Accuracy } \\
(\%)\end{array}$ & Error & $\begin{array}{c}\text { Accuracy } \\
(\%)\end{array}$ & \\
\hline 0 & \begin{tabular}{|l|}
43 \\
\end{tabular} & \begin{tabular}{|l|}
0.0183 \\
\end{tabular} & \begin{tabular}{|l|}
98.17 \\
\end{tabular} & 0.1628 & \begin{tabular}{|l|}
83.72 \\
\end{tabular} & 90.945 \\
\hline 1 & 39 & 0.0249 & 97.51 & 0.1711 & 82.89 & 90.200 \\
\hline 2 & 38 & 0.0282 & 97.18 & 0.1777 & 82.23 & 89.705 \\
\hline 3 & 34 & 0.0498 & 95.02 & 0.1877 & 81.23 & 88.125 \\
\hline 4 & 28 & 0.0847 & 91.53 & 0.1877 & 81.23 & 86.380 \\
\hline 5 & 24 & 0.1113 & 88.87 & 0.2093 & 79.07 & 83.970 \\
\hline 6 & 22 & 0.1312 & 86.88 & 0.2309 & 76.91 & 81.895 \\
\hline 7 & 20 & 0.1528 & 84.72 & 0.2342 & 76.58 & 80.650 \\
\hline 8 & 18 & 0.1777 & 82.23 & 0.2658 & 73.42 & 77.825 \\
\hline 9 & 12 & 0.2608 & 73.92 & 0.2924 & 70.76 & 72.340 \\
\hline 10 & 2 & 0.4485 & 55.15 & 0.4551 & 54.49 & 54.820 \\
\hline 11 & 1 & 0.5000 & 50.00 & 0.4983 & 50.17 & 50.085 \\
\hline
\end{tabular}

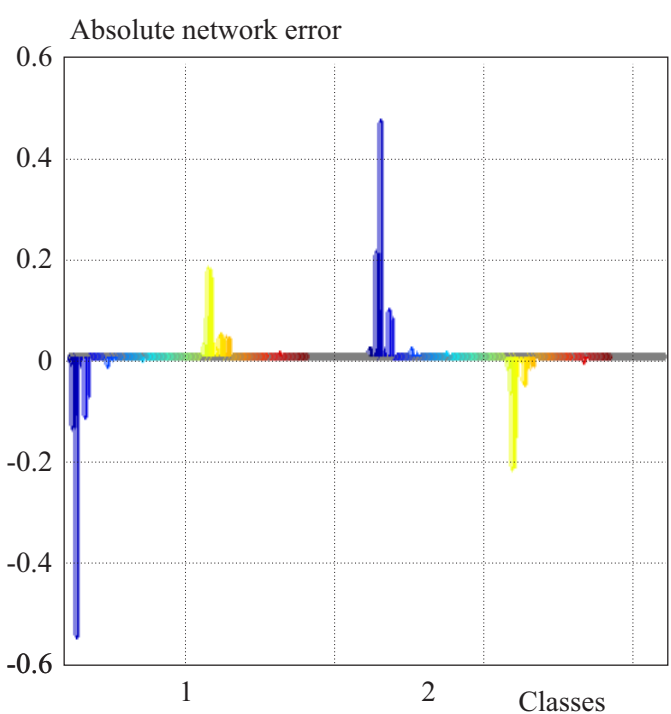

Fig. 4. 3d presentation of absolute network errors

Table 4. Matrix of correct and incorrect classifications for predictive decision model at analysis of $\mathrm{M} / \mathrm{M} / 25$ and $\mathrm{M} / \mathrm{M} / 25 / \mathrm{k}$ chains

\begin{tabular}{lcc}
\hline $\mathrm{M} / \mathrm{M} / \mathrm{c}$ & 295 & 6 \\
$\mathrm{M} / \mathrm{M} / \mathrm{c} / \mathrm{k}$ & 5 & 298 \\
\hline
\end{tabular}

class 1 and $100 \%$ for output class 2; Precisions $100 \%$ and $98.2 \%$, respectively for class 1 and class 2 and Total accuracy $99.2 \%$.

$3 \mathrm{D}$ representation of the absolute network errors about the synthesized network model is given in Fig. 4. The errors represent the differences between the calculated and the theoretical expected network results. Their variation is limited to the range of minimum and maximum val- ues, respectively -0.5627 and 0.4761 . The errors reflect the correct and incorrect classified samples.

\section{Synthesis of Markov chain identification model based on decision tree method}

A study of applicability of the decision tree method in building a model for predicting the membership of the teletraffic Markov chains was made. The following labels of input independent predictive variables at construction of classification structure are used:

- Cust. ID label CID; Fig. 5.

- Arrival Time - label AT;

- Server ID - label SID;

- Exit System - label ES.

Each of them is evaluated as an informative feature and is not excluded at decision tree generation. The Generated decision tree defines rules in a hierarchical structure in the form of a sequence of logical constructions "if-then" based on a classification of the target teletraffic systems.

Procedures related to reduce the decision tree model by removing of structural nodes and examine the quality of classification by resubstitution and cross-validation techniques are applied at different pruning levels. At resubstitution method $100 \%$ of dataset are used for training and test of trees while a cross-validation process $75 \%$ for training and $25 \%$ for testing of validation models, respectively. The results of the quality analysis are summarized in Tab. 3. A tendency to increase the error and decrease the accuracy is observed at both techniques with increasing of pruning level. For the model in Fig. 5, minimum errors 0.0183 and 0.1628 and maximum accuracies $98.17 \%$ and $83.72 \%$ were achieved. After removing the last possible nodes at the pruning level 11, the biggest errors were 


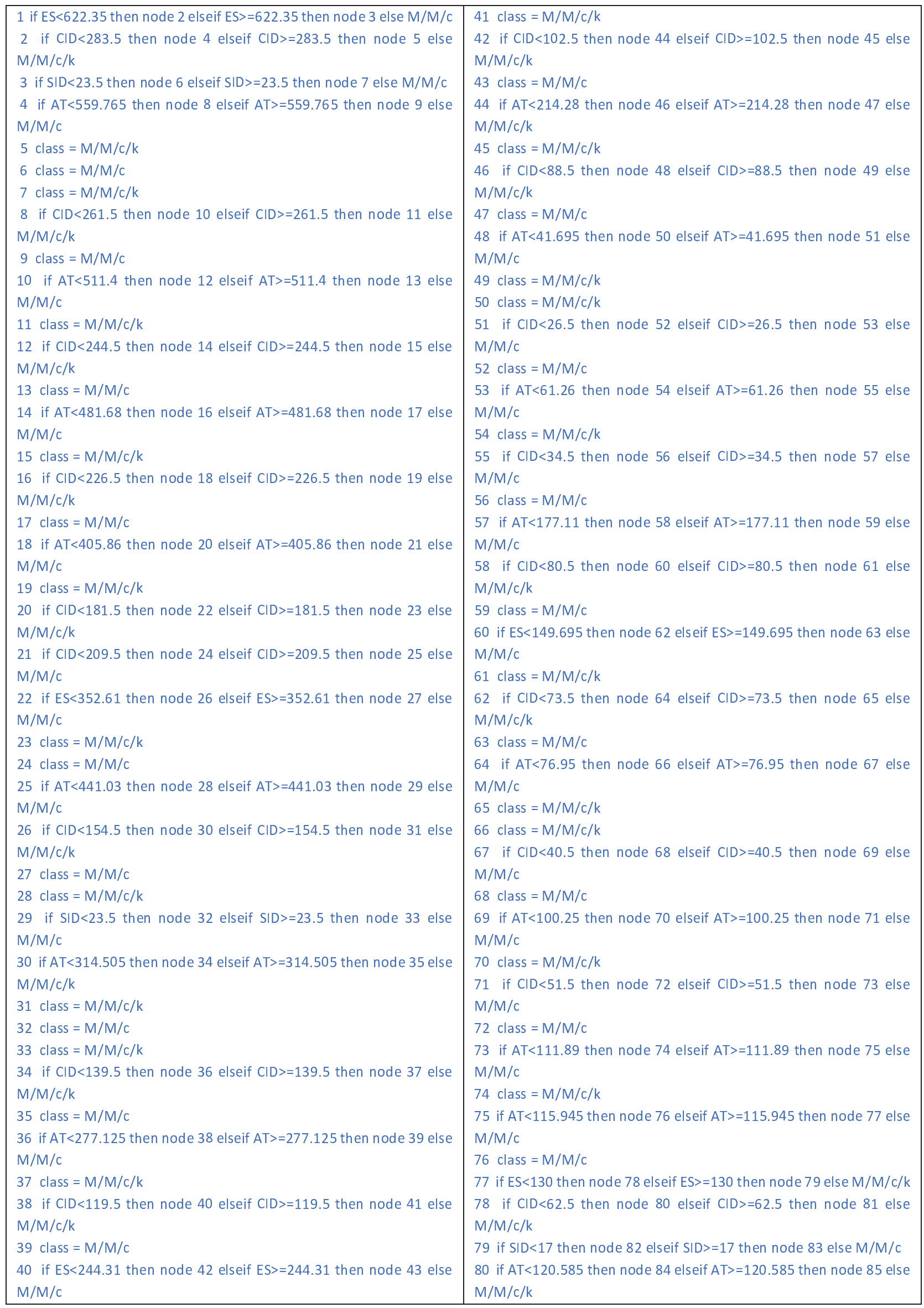

Fig. 5. Generated model "decision tree" for identification of systems $M / M / 25$ and $M / M / 25 / k$ at pruning level 0 
0.5000 and 0.4983 , respectively $50.00 \%$ and $50.17 \%$ accuracies. The approximately expected best and worst accuracies in telegtraffic identification were obtained $90.945 \%$ and $50.085 \%$, respectively. Based on the results of the analysis, a predictive model at pruning level 0 is synthesized for teletraffic system identification.

Table 4 represents the classification matrix for the synthesized decision tree model from which it follows that 295 and 296 samples are correctly classified for each output group. The incorrect classifications are total $11-6$ samples from class No1 are assigned to class No2 and 5 samples corresponding to the second classification group are classified to the first output group.

\section{Conclusion}

An innovative approach to identifying Markov's teletraffic chains by means of multilayer backpropagation neural network and decision tree structure is proposed. The synthesis processes of the identification models are performed on the basis of a step quantitative analysis of accepted parametric criteria - mean squared error and accuracy for ANN, and accuracies at re-substittion and cross-validation techniques for DT. The obtained best results give optimistic grounds and a prerequisite for deepening the research as search and combining of other methods, algorithms and apparatuses in the Markov chain type identification as well as improve the predictive processes of the teletraffic system identification.

\section{REFERENCES}

[1] P. Sokólski and T. Rutkowski, "Hybrid of Neural Networks and Hidden Markov Models as a modern approach to speech recognition systems", Pomiary Automatyka Robotyka, vol.17, no.2, pp.449-455, 2013.

[2] M. Mustafa, T. Allen and K. Appiah, "A comparative review of dynamic neural networks and hidden Markov model methods for mobile on-device speech recognition", Springer, Neural Comput \& Applic, pp.1-9, 2017.

[3] M. Yang, W. Tu, W. Yin and Z. Lu, "Deep Markov Neural Network for Sequential Data Classification", Proceedings of the 53rd Annual Meeting of the Association for Computational Linguistics and the 7th International Joint Conference on Natural Language Processing, pp.32-37, 2015.

[4] J. Bernstein, I. Dasgupta, D. Rolnick and H. Sompolinsky, "Markov Transitions between Attractor States in a Recurrent Neural Network", AAAI Spring Symposium Series, Science of Intelligence: Computational Principles of Natural and Artificial Intelligence, pp.1-5, 2017.

[5] D. Lowd and J. Davis, "Learning Markov Network Structure with Decision Trees", IEEE International Conference on Data Mining, Sydney, NSW, pp.334-343, 2010.

[6] D. Lowd and J. Davis, "Improving Markov Network Structure Learning Using Decision Trees", Journal of Machine Learning Research (JMLR), no.15, pp.501-532, 2014.

[7] B. Lakshminarayanan, D. Roy and Y. The, "Top-Down Particle Filtering for Bayesian Decision Trees", Proceedings of the 30th
International Conference on Machine Learning, Atlanta, Georgia, USA, JMLR: W\&CP, vol.28, pp.1-9, 2013.

[8] D. Bacciu, "Hidden Tree Markov Networks: Deep and Wide Learning for Structured Data", Proceedings of the IEEE Symposium Series on Computational Intelligence (IEEE SSCI), Honolulu, HI, pp.1-8, 2017.

[9] E. Ivanova, T. Iliev, Gr. Mihaylov and R. Rashkov, "Significant Simulation Parameters for RESTART/LRE Method in Teletraffic Systems of Network of Queues", IN: Automation Control Theory Perspectives in Intelligent Systems, Springer, pp.325-336, 2016.

10] E. Ivanova, T. Iliev, Gr. Mihaylov, I. Stoyanov, F. Tsvetanov, E. Otsetova and D. Radev, "Computer Modeling and Simulation of Teletraffic Models for 5G Networks", 38th International Convention on Information and Telecommunication Technology, Electronics and Microelectronics-MIPRO, Croatia, pp.479-482, 2015.

Received 10 July 2018

Ivelina Balabanova was born in Gabrovo, Bulgaria in 1977. She received the Master degree in electrical electronics engineering from the Technical University of Gabrovo, Bulgaria, in 2001. She is pursuing the $\mathrm{PhD}$ degree in electronics engineering at the department of Electronics Engineering in Technical University of Gabrovo. Currently, she is a senior assistant in the Department of the Communication Equipment and Technologies, Technical University of Gabrovo, Bulgaria. Major Fields of Scientific Research: Communication Networks and Systems, ptical Communications, Teletraffic Systems, Automation of Design and Modeling.

Georgi Georgiev was born in Pleven, Bulgaria in 1983. He received the BS degree in automatic engineering from the Technical University of Gabrovo, Bulgaria in 2007. He is pursuing the MS degree in electrical and electronics department, Technical University of Gabrovo, Bulgaria. Major Fields of Scientific Research: Measurement and control of air environment parameters in closed premises.

Stanimir Sadinov was born in Veliko Tarnovo, Bulgaria in 1969. He received the MS degree in electronics engineering from the Technical University of Gabrovo, Bulgaria in 1994. $\mathrm{He}$ is pursuing the $\mathrm{PhD}$ degree in communication engineering at the department of Telecommunications Engineering in Technical University of Gabrovo. He is currently Head of Department of the Communication Equipment and Technologies, Technical University of Gabrovo, Bulgaria. Major Fields of Scientific Research: Communication Networks and Systems, Digital Television Broadcasting, Mobile Communications.

Stela Kostadinova was born in Sofia, Bulgaria, in 1965. She received the MSc degree in electrical engineering from the Moscow Technical University of Communications and Informatics, Moscow, Russia, in 1988. Since joining Bulgarian Telecommunication Company (BTC) in 1989 she has been involved in many international and national telecommunication projects in optimization, design and management of architectures for SDH and WDM optical transport networks. For 10 years she led the Fiber-optic and Digital Transmission Systems Department in BTC. In 2011, she joined the Department of Telecommunications, Technical University of Varna, as an Assistant Professor. Her current research interests include telecommunication networks, optical telecommunications, next generation networks, neural networks, and quality of service. 